\title{
Selection of integral functions for normal mode analysis in topology optimization
}

\author{
Liyong Tong ${ }^{1, a}$ and Quantian Luo ${ }^{1, b}$ \\ ${ }^{1}$ School of Aerospace, Mechanical and mechatronic Engineering, The University of Sydney, Sydney, \\ NSW 2006, Australia \\ aliyong.tong@sydney.edu.au, ㅁuantian.luo@sydney.edu.au
}

Keywords: normal mode analysis, topology optimization, moving iso-surface

\begin{abstract}
This article investigates topology optimization for normal mode analysis using a moving iso-surface threshold method. Fundamental natural frequency needs to be calculated for many engineering structures and maximizing its value is an interesting topic in topology optimization. Optimal design for the maximum fundamental frequency may appear to be a trivial issue or impractical design. Reinforcements by introducing non-designable elements and non-structural mass or concentrated mass are often used. In this article, these issues will be solved by choosing an appropriate $\Phi$ function that is an integral function in the moving iso-surface threshold method. The proposed $\Phi$ function is expressed as strain and kinetic energy densities for a series of normal modes. By selecting the energy densities of different mode shapes, optimal topologies to maximize structural fundamental frequency are studied.
\end{abstract}

\section{Introduction}

Topology optimization is a broad range of disciplines covering mathematics, multi-field physics, computer science and structural engineering. It has attracted considerable interests in a field of structural design to achieve marvellous structural performance by optimizing material distributions. In structural design, increasing fundamental natural frequency for a given design domain is often required to avoid or promote vibration resonance.

Optimal design to maximize the $1^{\text {st }}$ order natural frequency has been studied by a number of authors using various topology optimization methods [1-6]. In this paper, a moving iso-surface threshold method (MIST) is used. Optimal design to maximize fundamental frequency may be trivial as it could be achieved by removing an entire structure [7]. To solve this trifling issue, reinforcements by specifying non-designable elements and/or adding non-structural mass can be used [4, 7]. In this article, this issue will be attempted by selecting appropriate integral functions used in MIST.

A number of topology optimization methods have been developed for optimal structural designs to obtain better structural performance for a specified design objective, such as, a homogenization method [8], solid isotropic material with penalisation (SIMP) method [9-11] with optimal criteria (OC) and moving asymptotes [12] algorithms, evolutionary structural optimization (ESO) [13] and a level set method [11, 14-17]. A moving iso-surface threshold method (MIST) [18, 19] was recently proposed for optimal design of minimum structural compliance or maximum stiffness and designs of gripping mechanism and pressurized cellular structures. Here, this method will be used to investigate an increase of fundamental natural frequency.

\section{A moving iso-surface threshold method for maximum fundamental natural frequency}

\subsection{A moving iso-surface threshold method}

In the MIST, a physical response is expressed using the $\Phi$ function that is evaluated at nodes in a finite element design domain. The level or threshold value of an iso-surface $S$ that depends on 
constraints is used to intersect the iso-surface $S$ with the function $\Phi$ surface to form a contour of the structural boundary. This method can be stated as [18, 19]:

$$
\begin{cases}\text { Maximum : } & f(t)=\int_{\Omega} F(x, t) h(t, \Phi(x)) d \Omega \\
\text { Subject to : } & \left\{\begin{array}{l}
g_{r}(t)=\int_{\Omega} G_{r}(x, t) h(t, \Phi(x)) d \Omega=0 \\
g_{s}(t)=\int_{\Omega} G_{s}(x, t) h(t, \Phi(x)) d \Omega \leq 0 \\
\end{array}(x)=\right.\text { response function }\end{cases}
$$

where $f(t)$ is the objective function; $g_{r}(t)$ and $g_{s}(t)$ are the governing and constraint equations; $F(x, t), G_{r}(x, t)$ and $G_{s}(x, t)$ are the relevant integral functions; $\Phi(x)$ is the response function and $t$ denotes the level or threshold; $h(x)$ is the Heaviside function defined by: $h(x)=1 \forall x \in\{\phi(x) \geq t\}$ or $h(x)=0 \forall x \in\{\phi(x)<t\}$.

\subsection{Finite element based normal mode analysis}

Motion equation of a structure with no damping for each mode in finite element formulations can be expressed as [20]:

$[\boldsymbol{K}]\left\{\boldsymbol{Y}_{\boldsymbol{i}}\right\}=\omega_{i}^{2}[\boldsymbol{M}]\left\{\boldsymbol{Y}_{\boldsymbol{i}}\right\} \quad(i=1,2,3, \ldots)$

where $[\boldsymbol{K}]$ and $[\boldsymbol{M}]$ are the stiffness and mass matrices; $\omega_{i}(i=1,2,3, \ldots)$ is the $i^{\text {th }}$ order frequency and $\left\{\boldsymbol{Y}_{\boldsymbol{i}}\right\}$ is its mode shape. In general, $[\boldsymbol{K}]$ and $[\boldsymbol{M}]$ are real symmetrical matrices and thus $\left\{\boldsymbol{Y}_{\boldsymbol{i}}\right\}(i=$ $1,2,3 \ldots)$ are orthogonal each other [20]:

$$
\left\{\boldsymbol{Y}_{i}\right\}^{T}[\boldsymbol{M}]\left\{\boldsymbol{Y}_{\boldsymbol{j}}\right\}=\left\{\begin{array}{cc}
0 & i \neq j \\
m_{i} & i=j
\end{array} ; \quad\left\{\boldsymbol{Y}_{\boldsymbol{\imath}}\right\}^{T}[\boldsymbol{K}]\left\{\boldsymbol{Y}_{\boldsymbol{j}}\right\}= \begin{cases}0 & i \neq j \\
k_{i} & i=j\end{cases}\right.
$$

where $m_{i}$ and $k_{i}$ is the generalized mass and stiffness. When an eigenvector for $i^{\text {th }}$ mode shape is normalized to result in a unit value of generalized mass $\left(m_{i}=1\right)$, one has:

$$
\left\{\boldsymbol{Y}_{\boldsymbol{l}}\right\}^{T}[\boldsymbol{K}]\left\{\boldsymbol{Y}_{\boldsymbol{i}}\right\}=\omega_{i}^{2}=k_{i} ; \quad\left\{\boldsymbol{Y}_{\boldsymbol{l}}\right\}^{T}[\boldsymbol{M}]\left\{\boldsymbol{Y}_{\boldsymbol{i}}\right\}=1 \quad(i=1,2,3 \ldots)
$$

In this case, total strain and kinetic energies for the $i^{\text {th }}$ mode can be calculated by [20]:

$$
\begin{array}{ll}
(S E)_{i}=\frac{1}{2}\left\{\boldsymbol{Y}_{\boldsymbol{i}}\right\}^{T}[\boldsymbol{K}]\left\{\boldsymbol{Y}_{\boldsymbol{i}}\right\} & (i=1,2,3 \ldots) \\
(K E)_{i}=\frac{1}{2}\left\{\dot{\boldsymbol{Y}}_{\boldsymbol{i}}\right\}^{T}[\boldsymbol{M}]\left\{\dot{\boldsymbol{Y}}_{\boldsymbol{i}}\right\}=\frac{1}{2} \omega_{i}^{2}\left\{\boldsymbol{Y}_{\boldsymbol{i}}\right\}^{T}[\boldsymbol{M}]\left\{\boldsymbol{Y}_{\boldsymbol{i}}\right\} & (i=1,2,3 \ldots)
\end{array}
$$

where $(S E)_{i}$ and $(K E)_{i}$ represent the total strain and kinetic energies; $\left\{\dot{\boldsymbol{Y}}_{i}\right\}$ denotes the mode shape velocity. By using Eqs. (4), (5a) and (5b), the $i^{\text {th }}$ order natural frequency can be calculated by: $\omega_{i}^{2}=2(S E)_{i}$ or $\omega_{i}^{2}=2(K E)_{i}$

$$
\begin{array}{ll}
\omega_{i}^{2}=2(S E)_{i} \text { or } \omega_{i}^{2}=2(K E)_{i} & (i=1,2,3 \ldots) \\
\omega_{i}^{2}=2 \int_{\Omega}(S E D)_{i} d \Omega \text { or } \omega_{i}^{2}=2 \int_{\Omega}(K E D)_{i} d \Omega & (i=1,2,3 \ldots)
\end{array}
$$

where $(S E D)_{i}$ and $(K E D)_{i}$ are the strain and kinetic energy densities for the $i^{\text {th }}$ order mode.

\subsection{Selection of integral functions to maximize fundamental natural frequency}

It is seen from Eq. (6b) that, the strain or kinetic energy density can be used as the integral function to maximize the $i^{\text {th }}$ order frequency. The $1^{\text {st }}$ order frequency can be generally expressed as: 


$$
\begin{aligned}
\omega_{1} & =\frac{2}{\omega_{1}}\left\{\left[1-\alpha_{K}-\sum_{l}\left(\alpha_{l s}+\alpha_{l k}\right)\right] \int_{\Omega}(S E D)_{1} d \Omega+\alpha_{K} \int_{\Omega}(K E D)_{1} d \Omega\right. \\
& \left.+\sum_{l}\left[\alpha_{l s}\left(\frac{\omega_{1}^{2}}{\omega_{l}^{2}}\right) \int_{\Omega}(S E D)_{l} d \Omega\right]+\sum_{l}\left[\alpha_{l k}\left(\frac{\omega_{1}^{2}}{\omega_{l}^{2}}\right) \int_{\Omega}(K E D)_{l} d \Omega\right]\right\}
\end{aligned}
$$

where $\alpha_{K}, \alpha_{l s}$ and $\alpha_{l k}$ are coefficients; $l \neq 1$. When the $i^{\text {th }}$ order frequency is to be maximized, the formulation similar to Eq. (7) can be derived accordingly.

\subsection{MIST implementation by using a MatLab program interfaced with commercial software}

On the basis of Eq. (7), the $\Phi$ function can be constructed by using a combination of strain and kinetic energy densities with different modes. In the present implementation using the MIST, strain and kinetic energy densities in element centres are computed by using MSC/NASTRAN and output to MatLab; their values at nodes are calculated by the Lagrange interpolation and then the MIST iterations similar to those in $[18,19]$ are conducted.

\section{Numerical results, verification and discussion}

\subsection{Numerical results and comparison}

Topology optimization to maximize $1^{\text {st }}$ order frequency of a beam fixed two ends shown in Fugure 1 was studied in [4] using a SIMP method. Its normalized geometric and material data are: $a=1$, $b=8, E=10^{7}, v=0.3$ and $\rho=1$, where $E, v$ and $\rho$ represent Young's modulus, Poisson's ratio and the mass density.

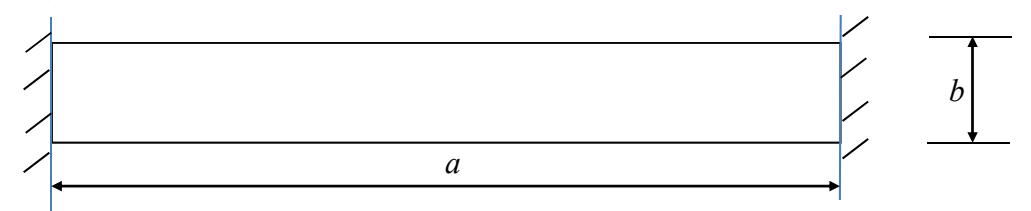

Figure 1 A beam-like structure fixed at two ends

By observing strain and kinetic energy distributions for a structure of Figure 1, the $\Phi$ function to maximize the $1^{\text {st }}$ order frequency in plane stress can be constructed by its strain and kinetic energy densities:

$\Phi_{j}=\left(1-\alpha_{K}\right)(S E D)_{1 j}+\alpha_{K}(K E D)_{1 j}$

where subscript $j$ denote the node numbers. When $\alpha_{K}=0$ and -0.5 , the obtained topologies for a volume fraction of 0.5 (mesh: $30 \times 240$ ) are shown in Figure 2.

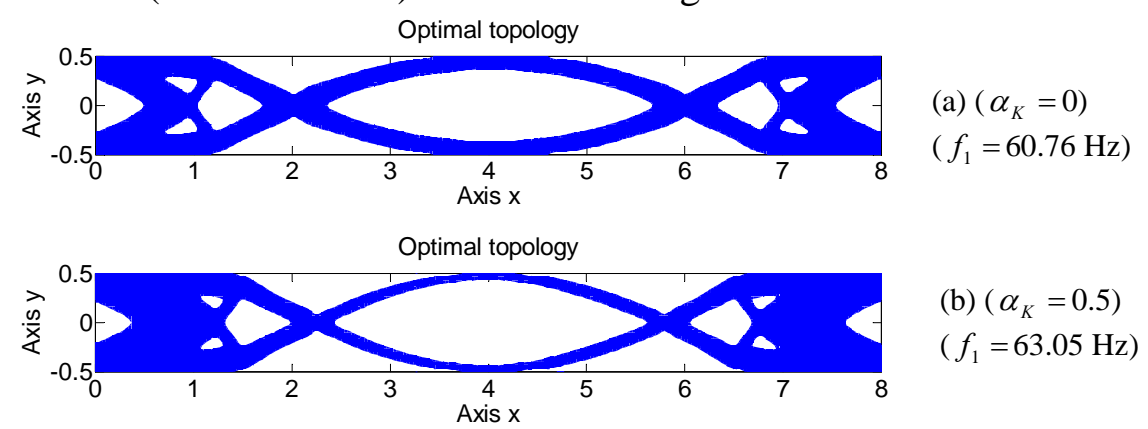

Figure 2 Optimal topologies for different integral functions

Figure 2 shows that higher fundamental frequency can be achieved by choosing a proper srain and kinetic energy density combinations. 
A canlitelever square plate with a length of 1 (m) is shown in Fig. 3 (a). $E=70$ GPa, $v=0.3$ and $\rho=2700\left(\mathrm{~kg} / \mathrm{m}^{3}\right)$. The optimal design for the maximum $1^{\text {st }}$ order frequency of this plate is trifling as it can be obtained by removing whole part from the free edge. The enforcement with the non-designable elements and/or concentrated mass have been used to obtain non-trivial optimal design in $[4,5]$. In this paper, the following $\Phi$ function is used in iterative computations to obtain the practical optimal topology with the maximum fundamental frequency:

$\Phi_{j}=\left(1+\alpha_{2 k}\right)(S E D)_{1 j}-\alpha_{2 k}(K E D)_{2 j}$

When $\alpha_{2 k}=-0.15$, the achieved optimal topology for a volume fraction of 0.5 (mesh: $40 \times 40$ ) is given in Figure 3(b) and the frequency variation versus iteration is illustrated in Figure 4.

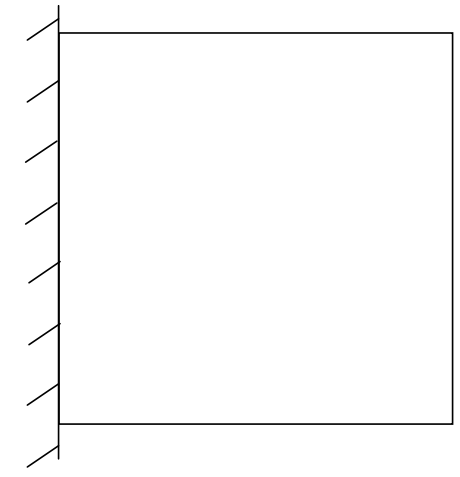

(a)

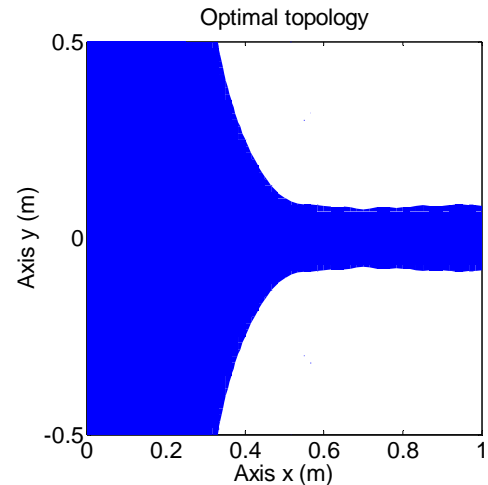

(b)

Figure 3 A cantlever plate (a) a square plate; (b) optimal design for the maximum $1^{\text {st }}$ order frequency

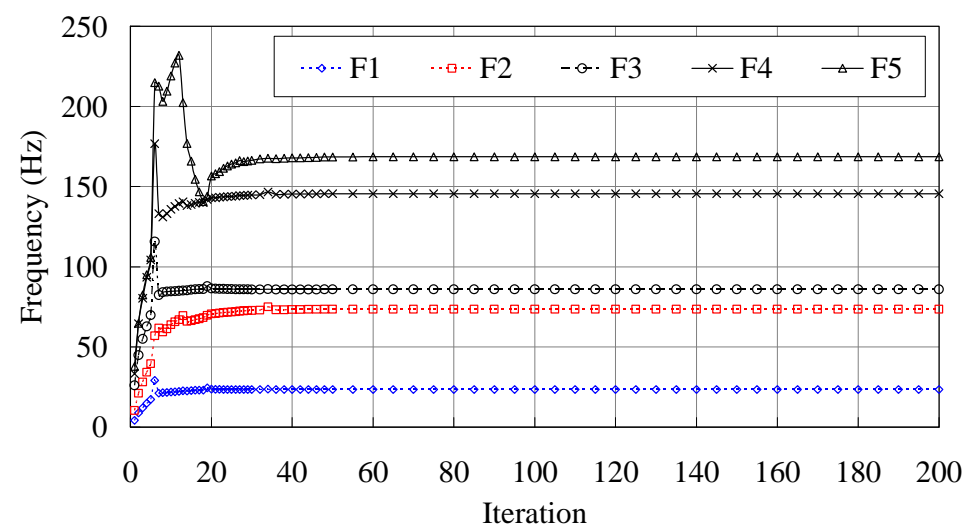

Figure 4 Frequency versus iteration for plate vibration ( $\mathrm{Fi}-$ the $i^{\text {th }}$ order frequency)

It is seen from Figure 4 that the frequencies for the $1^{\text {st }}-5^{\text {th }}$ normal modes are almost the same after the sixty iterations. The converged $1^{\text {st }}$ frequency is $23.48(\mathrm{~Hz})$.

\subsection{Validation, comparison and soft material effects}

It is noted that optimal topology in Figure 2(a) and 2(b) are similar to those obtained in [4] and [5], where the SIMP method was used. For solid material distributions given in Figures 2(a) and 3(b), finite element results output by NASTRAN are illustrated in Figures 5(a) and 5(b).

When the solid material distribution in Figure 2(a) is imported to PATRAN, the $1^{\text {st }}$ order frequency computed by NASTRAN is $61.61(\mathrm{~Hz})$, whis is slightly larger than that $(60.76 \mathrm{~Hz})$ with void and soft materials for Figure 2(a). That is, soft materials have little influence on frequency of this structure. However, the 1st order frequency given in Figure 4 and Figure 5(b) is 23.48 and $15.47(\mathrm{~Hz})$, respectively. Therefore, soft materials can significantly increase fundamental frequency of plate vibrations. 

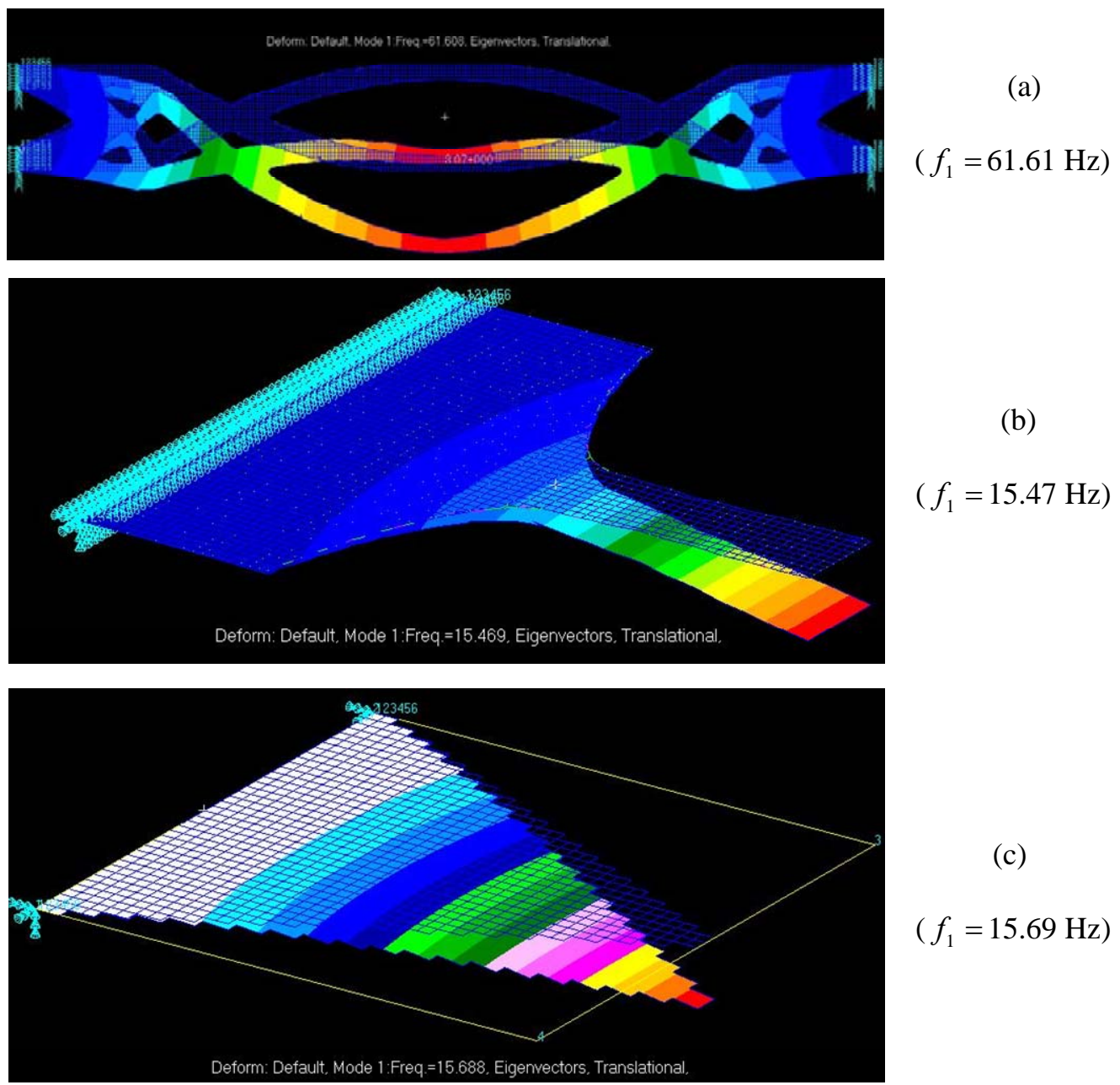

Figure 5 The $1^{\text {st }}$ mode shape (a) for Figure 2; (b) for Figure 4; (a) predicted by Niu et al. [5]

To obtain optimal design for the maximum fundamental frequency of a plate in Figure 4(a), a concentrated mass has been applied to the middle of a free edge in $[4,5]$ and almost the same topology has been obtained in [4] and [5]. Figure 5(c) illustrates the $1^{\text {st }}$ order mode shape predicted by using the solid material distribution given in [5]. The fundamental frequencies given in Figures 5(b) and 5(c) are 15.47 and $15.69(\mathrm{~Hz})$, respectively. Therefore, present topology optimization using the MIST to maximize fundamental frequency is verified for plate vibration. It is also seen that topology optimization to maximum fundamental frequency can be alternatively achieved by choosing appropriate $\Phi$ functions rather than using enforcement approaches or adding non-structure masses.

\section{Concluding remarks}

A moving iso-surface threshold method was used to study optimal design for normal mode analysis. Integral functions to maximize fundamental frequency are derived. Numerical results show that the fundamental frequency can be significantly increased by selecting better integral functions.

Void and soft materials have little effect on frequency of in-plane vibration but affect out-of-plane vibrations of plate structures considerably. Optimization for the selection of integral functions and analysis of the soft material influences are to be further investigated.

\section{Acknowledgement}

The authors are grateful for the support of the Australian Research Council via Discovery-Project Grants (DP110104123). 


\section{Reference}

1. Pedersen, N.L., Maximization of eigenvalues using topology optimization. Structural and Multidisciplinary Optimization, 2000. 20(1): 2-11.

2. Tcherniak, D., Topology optimization of resonating structures using SIMP method. International Journal for Numerical Methods in Engineering, 2002. 54(11): 1605-1622.

3. Jensen, J.S. and Pedersen, N.L., On maximal eigenfrequency separation in two-material structures: the $1 D$ and $2 D$ scalar cases. Journal of Sound and Vibration, 2006. 289(4-5): 967-986.

4. Du, J. and Olhoff, N., Topological design of freely vibrating continuum structures for maximum values of simple and multiple eigenfrequencies and frequency gaps. Structural and Multidisciplinary Optimization, 2007. 34(2): 91-110.

5. Niu, B., Yan, J. and Cheng, G., Optimum structure with homogeneous optimum cellular material for maximum fundamental frequency. Structural and Multidisciplinary Optimization, 2009. 39(2): 115-132.

6. Ma, Z.-D., Cheng, H.-C. and Kikuchi, N., Structural design for obtaining desired eigenfrequencies by using the topology and shape optimization method. Computing Systems in Engineering, 1994. 5(1): 77-89.

7. Bendsoe, M.P. and Sigmund, O., Topology Optimization: Theory, Methods and Applications2003, Berlin ; New York: Springer.

8. Bendsoe, M.P. and Kikuchi, N., Generating optimal topologies in structureal design using a homogenization method. Computer Methods in Applied Mechanics and Engineering, 1988. 71(2): 197-224.

9. Bendsøe, M.P., Optimal shape design as a material distribution problem. Structural optimization, 1989. 1(4): 193-202.

10. Mlejnek, H.P., Some aspects of the genesis of structures. Structural optimization, 1992. 5(1-2): 64-69.

11. Zhou, M. and Rozvany, G.I.N., The COC algorithm, Part II: Topological, geometrical and generalized shape optimization. Computer Methods in Applied Mechanics and Engineering, 1991. 89(1-3): 309-336.

12. Svanberg, K., The method of moving asymptotes-a new method for structural optimization. International Journal for Numerical Methods in Engineering, 1987. 24(2): 359-373.

13. Xie, Y.M. and Steven, G.P., A simple evolutionary procedure for structural optimization. Computers \& Structures, 1993. 49(5): 885-896.

14. Sethian, J.A., Fast marching methods. Siam Review, 1999. 41(2): 199-235.

15. Wang, M.Y., Wang, X.M. and Guo, D.M., A level set method for structural topology optimization. Computer Methods in Applied Mechanics and Engineering, 2003. 192(1-2): 227-246.

16. Luo, J.Z., Luo, Z., Chen, L.P., Tong, L.Y. and Wang, M.Y., A semi-implicit level set method for structural shape and topology optimization. Journal of Computational Physics, 2008. 227(11): 5561-5581.

17. Allaire, G., Jouve, F. and Toader, A.-M., A level-set method for shape optimization. Comptes Rendus Mathematique, 2002. 334(12): 1125-1130.

18. Tong, L.Y. and Lin, J.Z., Structural topology optimization with implicit design variable-optimality and algorithm. Finite Elements in Analysis and Design, 2011. 47(8): 922-932.

19. Vasista, S. and Tong, L.Y., Design and Testing of Pressurized Cellular Planar Morphing Structures. AIAA Journal, 2012. 50(6): 1328-1338.

20. Cook, R.D., Malkus, D.S. and Plesha, M.E., Concepts and applications of finite element analysis. 4th ed2001, 4th edition. New York: Wiley, c2001. 